\title{
二磷酸腺苷核糖转移酶抑制剂 对匹莱䨣素的抗癌协同作用
}

\author{
陈海英潘启 超 \\ (中山医科大学肿㿔研究所抗癌药物研究室,广州)
}

二磷酸腺苷核糖转移酶 (ADPRT) 催化辅酶 I(NAD) 分子上的 ADP-核糖组分转栘到 蛋白质或另一个 ADP-核糖分子上形成多聚体 $(A D P-R)_{n}$. 这一过程与细胞的多种生物学功 能有关. 近年来国外一些研究报道该酶的抑制剂在抑制 DNA 修复的同时还能加强某些抗 癌药物如链腿露素 ${ }^{[1]}$ 、博莱霉素 ${ }^{[2-4]}$ 、亚硝腿类化合物 ${ }^{[9]}$ 和顺铂 ${ }^{[6]}$ 的抗癌作用，引起人们的关注， 为发展高效低毒的化疗途径提供了新的希望. 我们曾在体外实验证明 ADPRT 抑制剂 $3 \mathrm{MB}$ 能够增强匹莱霉素的体外细胞毒作用 ${ }^{[7]}$ ，在此基础上我们进一步观察了它们的体内作用. 皆 在为其推广到临床应用提供更充分的实验证据.

\section{一、材料和方法}

1.小鼠非纯种鼠，体重 $20 \mathrm{~g} \pm 2 \mathrm{~g}$, 雌雄兼有.

2. 药物匹莱霉素 (Peplomycin PEP) 日本化药公司产；3-甲氧基苯甲酰胺 (3-Methoxybenzamide，3MB）美国 Aldrich 化学公司产；3-氨基苯甲酰胺 (3-Aminobenzamide，3AB) 日本东京化成工业株式会社生产; 烟酰胺 (Nicotinamide, NA) 广州化学试剂厂生产. 上述 药物均在实验前以生理盐水溶解.

3. 实验方法 实验取接种后 7-8 天生长良好的实体型小鼠肝癌. 常规皮下接种,将动物 按雌雄体重随机分组，接种 24 小时后开始腹腔给药,连续 9 天, 停药 24 小时后处死小鼠, 秤 㾴重, 计算抑瘤率。

\section{二、实 验 结 果}

体内抑瘤试验共进行 7 次,除了重复验证 PEP $(1.5 \mathrm{mg} / \mathrm{kg} / \mathrm{d})$ 和 $3 \mathrm{MB}(1 \mathrm{mM} / \mathrm{kg} / \mathrm{d})$ 的协 同作用外,还分别观察了不同剂量的 PEP 和 $3 \mathrm{MB}$ 对协同作用的影响, 以及不同用药方案协 同作用的差异，并对三种抑制剂的协同作用进行了比较.

从表 1 可见 $3 \mathrm{MB} 1.5 \mathrm{mg} / \mathrm{kg}$ 每天 $1-2$ 次没有表现出抑瘤作用（与对照组相比 $P$ 值均大 于 0.05), 但能明显增强 PEP 的抑瘤作用. 如以单用 PEP 的抑瘤率为 1 , 加用 $3 \mathrm{MB}$ 后分别 为 $1.58,1.61,1.64,1.32,1.77,1.45$ 和 1.72. 抑瘤率的增加比值较为恒定, 具有重复性. $3 \mathrm{MB}$ $1 \mathrm{mg} / \mathrm{kg} / \mathrm{d}$ 与 PEP $1.5 \mathrm{mg} / \mathrm{kg} / \mathrm{d}$ 合用，抑㿔率能达到单用 $P E P ~ 3 \mathrm{mg} / \mathrm{kg} / \mathrm{d}$ 的水平 (见实验 3 和 4), 也就是说加用 $1 \mathrm{mM} / \mathrm{kg}$ 的 $3 \mathrm{MB}$ 相当于 PEP 的剂量增加 2 倍的作用.

实验观察了三种用药方案对协同作用的影响，(i) PEP 与 $3 \mathrm{MB}$ 同时联合用药; (ii) PEP 用药 2 小时后给予 $3 \mathrm{MB}$; (iii) PEP 与 $3 \mathrm{MB}$ 同时联合用药 2 小时后追加一次 $3 \mathrm{MB}$. 三种用

本文 1986 年 4 月 5 日收到。 
药方案其抑沺率与单用 PEP 相比, 差异均有显著性. 其中以后一种方案效果最佳, 前后联合 用药效果比同时联合用药稍差 (见实验 2 和 4).

表 1 ADPRT 抑制剂与 PEP 对小鼠訮瘁的作用

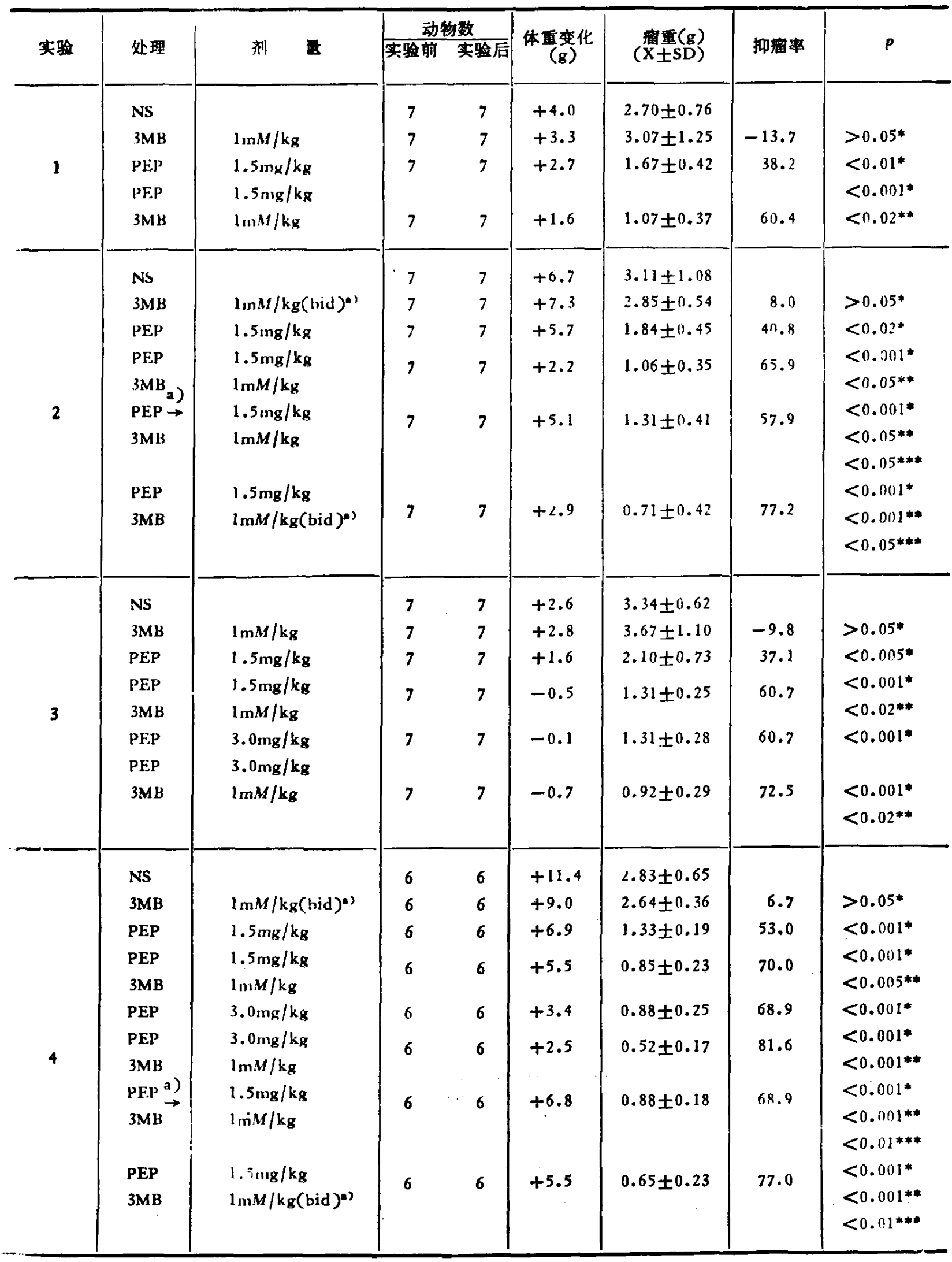


续表 1

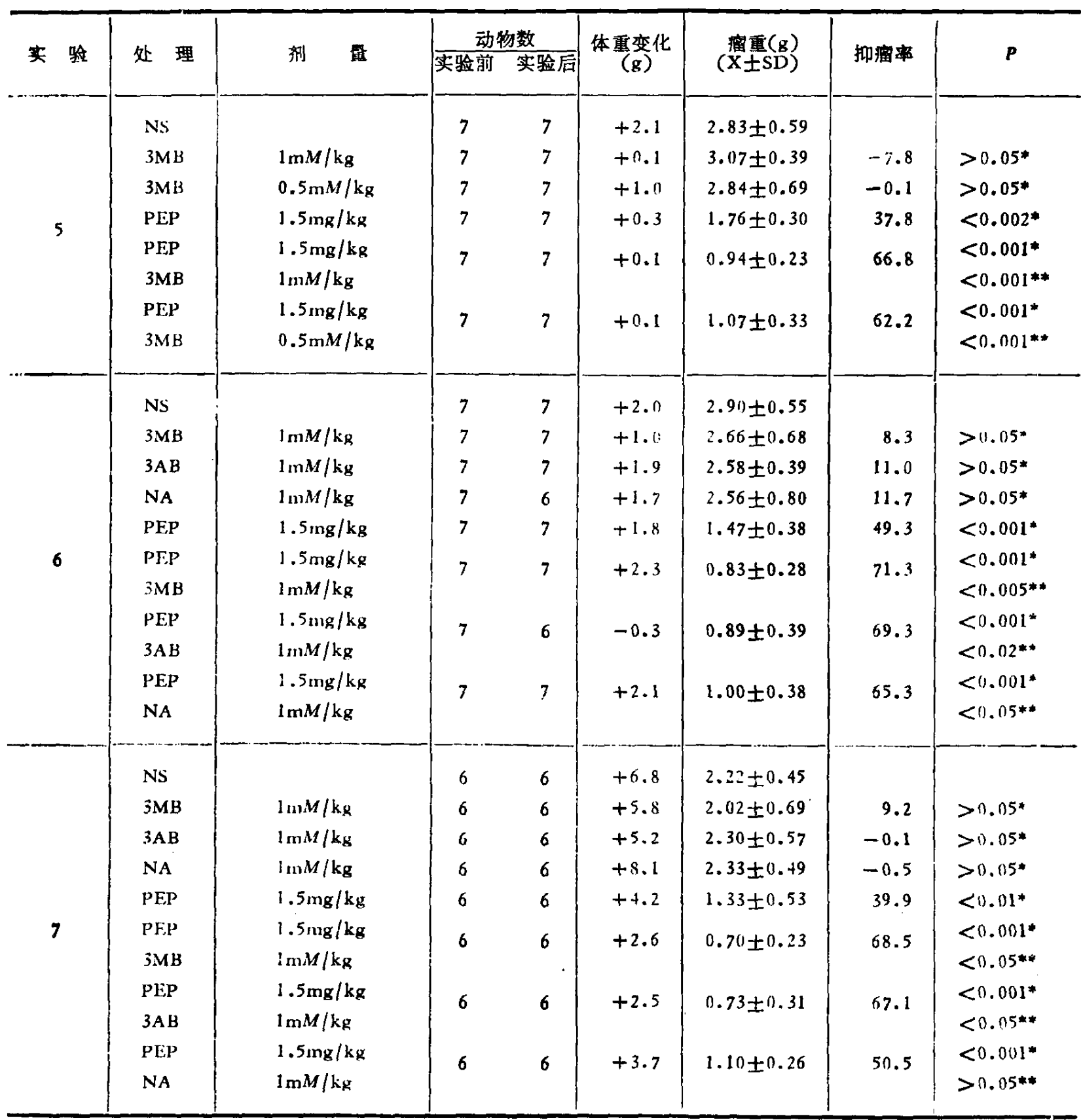

* 与对照组比较; ** 与单用 PEP 比较; *** 与 PEP + 3MB 比较; a) 首次用药 2 小时后给予第 2 次药物.

对三种抑制剂 $3 \mathrm{MB}, 3 \mathrm{AB}$ 和 $\mathrm{NA}$ 作用进行比较发现 在同样剂量下, $3 \mathrm{MB}$ 和 $3 \mathrm{AB}$ 对 PEP 的协同抗瘤作用较强，两者作用相当,而 NA 较弱，三种抑制剂本身单独应用均未发现 有抗癯作用 (见实验 6 和 7)。

$3 \mathrm{MB}$ 对 PEP 急性毒性影响见表 2, 3MB 没有增加 PEP 的致死性. 从抗瘤试验过程中 动物体重变化看, $3 \mathrm{MB}$ 也没有明显增加 PEP 的毒性作用.

\section{三、讨 论}

我们的实验证明了 $3 \mathrm{MB}$ 在不明显增加小鼠毒性的剂量下能明显增加 PEP 对小鼠肝癌的 抑制作用. 近年来关于 ADPRT 抑制剂与细胞毒药物的协同杀伤作用已有不少报道, 日本的 
表 $23 \mathrm{MB}$ 对 PEP 急性毒性的影响

\begin{tabular}{|c|c|c|c|c|c|c|c|c|}
\hline \multirow{2}{*}{ 处 理 } & \multicolumn{3}{|c|}{ 存 } & & 鼠 & \multicolumn{2}{|c|}{ 数 } & \multirow[b]{2}{*}{ 第7天 } \\
\hline & 第 0天 & 第 1 天 & 第 2 天 & 第 3 天 & 第 4天 & 第 5 天 & 第 6 天 & \\
\hline NS & 7 & 7 & 7 & 7 & 7 & 7 & 7 & 7 \\
\hline $3 \mathrm{MB} 1 \mathrm{mM} / \mathrm{kg}$ & 7 & 7 & 7 & 7 & 7 & 7 & 7 & 7 \\
\hline PEP $75 \mathrm{mg} / \mathrm{kg}$ & 7 & 7 & 7 & 7 & 7 & 7 & 7 & 7 \\
\hline $\begin{cases}\text { PEP } & 75 \mathrm{mg} / \mathrm{kg} \\
3 \mathrm{MB} & 1 \mathrm{~m} M / \mathrm{kg}\end{cases}$ & 7 & 7 & 7 & 7 & 7 & 7 & 7 & 7 \\
\hline PEP $100 \mathrm{mg} / \mathbf{k g}$ & 7 & 7 & 5 & 0 & 0 & 0 & $\mathbf{0}$ & 0 \\
\hline $\begin{cases}\text { PEP } & 100 \mathrm{mg} / \mathrm{kg} \\
3 \mathrm{MB} & 1 \mathrm{mM} / \mathrm{kg}\end{cases}$ & 7 & 6 & 4 & 2 & 1 & 1 & 1 & 0 \\
\hline
\end{tabular}

Sakamoto 发现另一种 ADPRT 抑制剂苯甲酰胺能明显增强博莱霉素对小鼠艾氏腹水癌的作 用,生命延长率从单用 BLM 的 $47 \%$ 提高到 $75 \%$ ，而在首次联合用药 3-4 天后追加一次苯 甲酰胺又能使生命延长率提高到 $97 \%$ ，我们的实验与此有相同的趋势. 提示在细胞毒药物 攻古肿瘤细胞后的一段时间内,追加 ADPRT 抑制剂能有效地增加对肿瘤细胞的杀伤，这可 能与 DNA 损伤修复动力学有关.

目前已知的 ADPRT 抑制剂有苯甲酰胺类、嘌呤类、烟酰胺类和胸苦四大类，以 ${ }^{3} \mathrm{H}-\mathrm{NAD}$ 掺入率为指标测定 ADPRT 的活性发现酶活性抑制作用以苯甲酰胺类最强 ${ }^{[8]}$. 我们实验证明 苯甲酰胺类抑制剂 $3 \mathrm{MB}$ 和 $3 \mathrm{AB}$ 对 PEP 的协同作用较烟酰胺强，与它们对 ADPRT 抑制 程度有相同趋势。

至于这种协同作用的机制估计与 DNA 修复有关. 因为已有充分的证据表明 ADPRT 参与了 DNA 的修复 ${ }^{[8]}$; 而 PEP 作为博莱荦素的第二代衍生物具有与博莱露素相似的切割 DNA 的能力 ${ }^{[9]}$. 而且有证据表明 PEP 所致的损伤在适宜条件下是可以修复的 ${ }^{[10]}$. 因此 $3 \mathrm{MB}$ 很可能是通过抑制 PEP 作用后的 DNA 修复过程发挥抗瘤协同作用.

PEP 正以其比博莱素抗瘤谱广、作用强、肺毒性低的优点受到日益重视，3MB 能在不 明显增加其毒性的前提下增强其抗癌作用。我们认为这是一种值得重视的联合化㡯方案,有 可能为临床提供一条高效低毒的化疗途径.

致谢：匹莱要素、3-甲氧基苯甲酰胺和 3-氮基苯甲酰胺由日本化药公司意赠,特此致谢。

\section{* 文獸}

[ 1 ] Schein, P. S. et al., Cancer Res., 27(1967), 2324-2332.

[2] Sakamoto, H. et al., J. Anribiotics, 36 (1983), 295-300.

[ 3] Kawamitsu, H. K. et al., J. Pharm. Dyn., 5(1982), 900-904.

[4] 陈关、采启趣, 药学报, 20(1985), 331-332.

[5] Berger, N A., Cancer Res, 42(1982), 4382-4386.

[ 6 ] Erlichman. C. et al., Proc. A. A. C. R. Abstract, 1984, 368.

[7] 陈海英、婇启趐, 中国药理学通报, 1985, 3: 20-22.

[ 8 ] Shall, S., Adv. Radia. Biol, 11(1984), 1-65.

[9] Takahashi, K. et al., J. Antibiotics, 32(1979), 36-40.

[10] Saito, T. et al., GANN, 75(1984), 147-151. 